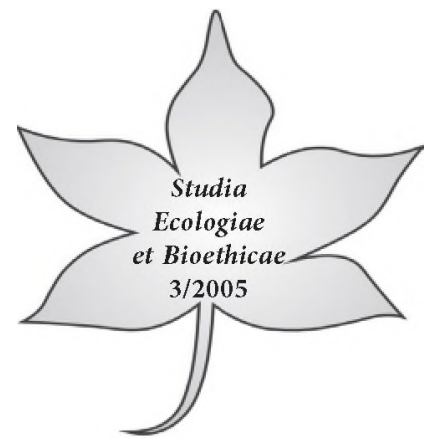

\title{
Zu den ökologischen Metaphern bei Paulus
}

Mehrmals hebt Paulus in seinen Briefen hervor, er sei $\alpha \pi o ́ \sigma \tau \lambda$ o $\varsigma$

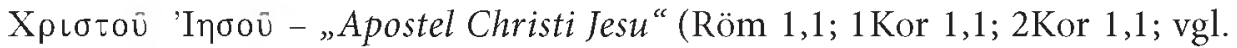
Röm 11,13;1Kor 9,1f; 15,9 u.a.). Als Apostel weiß er sich erwählt, berufen und gesandt das Evangelium Gottes zu verkündigen (Röm 1,1.9;15,16;1 Kor 1,17; 9,16; Gal 1,15f; 2,7)1. Im Präskript des Römerbriefes $(1,1)$ stellt sich Paulus der römischen Gemeinde, die er nicht gegründet hat, vor: $\Pi \alpha u \bar{\lambda} \lambda \circ \varsigma$

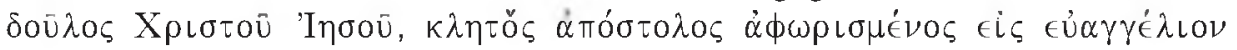
Ө૯ôิ - „Paulus, Knecht Christi Jesu, berufener Apostel, ausgesondert für das Evangelium Gottes". Damit will er sein Selbstverständnis als Apostel umschreiben und seine Autorität hervorheben ${ }^{2}$. In Hinsicht auf den Auftrag, den Paulus vom Auferstandenen Christus empfangen hat, schreibt er direkt, dass er

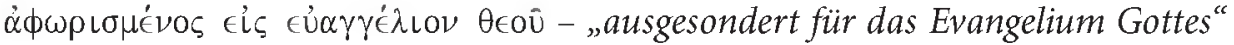

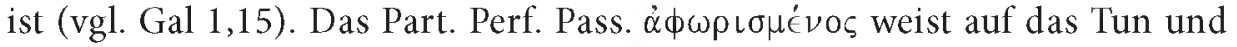

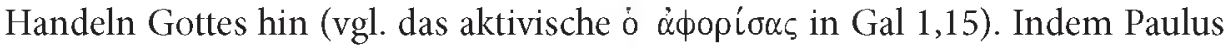
hier von seiner "Aussonderung" spricht, unterstreicht er, dass seine Berufung - wie die der Propheten - als göttliche Erwählung zu verstehen ist ${ }^{3}$. Das Ziel

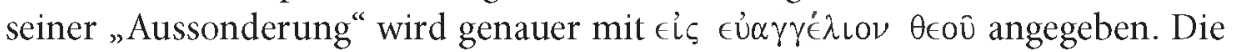
"Aussonderung von Gott ist also zunächst Zuordnung zum Evangelium. Zuordnung zum Evangelium heißt aber Zuordnung zu Gott. Die in der Aussonderung liegende Absonderung von "Menschen, Verhältnissen und Tätigkeiten «... ist gleichzeitig auch eine Zuordnung zu ihnen. Daß dieser Auftrag ein Paulus zugesprochenes Wort ist..., bestimmt auch sein Verhältnis zu Rom: seine Würde ist die des Evangeliums“" Die „Aussonderung“ ist also bezogen auf

Ausführlich dazu A.J. Najda, Der Apostel als Prophet. Zur prophetischen Dimension des paulinischen Apostolats (EHS XXIII/784), Frankfurt am Main - Berlin - Bern - Bruxelles - New York - Oxford - Wien 2004, 31-71.

2 Vgl. J. Zmijewski, Paulus - Knecht und Apostel Jesu Christi. Amt und Amtsträger in paulinischer Sicht, Stuttgart 1986, 38.

3 Vgl. u.a. U. Wilckens, Der Brief an die Römer. 1. Teilband. Röm 1 - 5 (EKK VI/1), Zürich - Einsiedeln - Köln - Neukirchen-Vluyn 1978, 63.

${ }^{4}$ O. Michel, Der Brief an die Römer $\left(\mathrm{KEK} \mathrm{IV}^{14}\right)$, Göttingen ${ }^{5} 1978,68 \mathrm{f}$. 
das Evangelium, dem Paulus zugeordnet ist ${ }^{5}$. Sie „meint die schon von Ewigkeit her feststehende göttliche Erwählung zur Verkündigung des Evangeliums ${ }^{\text {“‘ }}$.

\section{Metaphern in den paulinischen Briefen}

In seiner Verkündigung greift Paulus häufig zu Bildern ${ }^{7}$. Er bedient sich verschiedener Motive und Vorstellungen aus der Pflanzen-, Tierund Menschenwelt, „um verständlich zu sein, um zu überzeugen oder zu beeindrucken “8. Am meisten gebraucht der Apostel in seinen Briefen Metaphern. Unter Metapher ist primär „ein Kontextphänomen“, d.h. „nie ein einfaches Wort“, sondern „immer ein - wenn auch kleines - Stück Text “9. Die konkrete Metapher entsteht dann im Text durch einen Vorgang der "Konterdetermination “" in dem "die tatsächliche Determination des Kontextes gegen die Determinationserwartung des Wortes gerichtet ist " ${ }^{\text {"11 }}$, was beim Hörer bzw. Leser einen - für die Metapher charakteristischen - „Überraschungseffekt" ${ }^{\text {12 }}$ zur Folge hat. Die Metaphern treten häufig in Gruppen und im Zusammenhang mit anderen auf. Die Gruppierungen von Metaphern lassen sich als „Bildfelder “13 beschreiben.

5 Vgl. E. Käsemann, An die Römer (HNT 8a), Tübingen 1973, 4; K. Kertelge, Der Brief an die Römer (GSL.NT 6), Düsseldorf ${ }^{2} 1983,21$.

6 D. Zeller, Der Brief an die Römer (RNT), Regensburg 1985, 34.

7 W. Straub, Die Bildersprache des Apostels Paulus, Tübingen 1937, 20-97, zählt bei Paulus folgende Bildformen auf: die Bildwörter, die bildhaften Redewendungen, die Vergleiche, die Metaphern, die Bildsprüche und die Gleichnisse. Vgl. auch M. Reiser, Sprache und literarische Formen des Neuen Testaments. Eine Einführung, Paderborn - München - Wien - Zürich 2001, $76 f$.

8 K. Seybold, Die Sprache der Propheten. Studien zur Literaturgeschichte der Prophetie, Zürich 1999, 13. Nach seiner Ansicht ist das Ziel der Propheten, „Vorstellungen zu vermitteln, die die Propheten selbst in Form von Schauungen erhalten haben. Dass sie dabei auch Vorstellungen übermitteln, die in begrifflicher Fixierung der theologischen Einsicht dienen, ist nur die natürliche Folge solcher Redeweise. Ein bestimmtes "Menschenbild « und ein "Gottesbild» zeichnen sich ab" (a.a.O. 14).

9 H. Weinrich, Semantik der Metapher, in: Folia Linguistica I(1967), 3-17, 5. Nach P.G. Müller, Lexikon exegetischer Fachbegriffe, Stuttgart - Kevelaer 1985, 173, ist die Metapher „Ersetzung eines Begriffs durch einen anderen, der mit ersterem einen Vergleichspunkt hat". Nach J. Wehrle, Metapher, in: Neues Bibel-Lexikon II, hg. von M. Görg - B. Lang, Zürich - Düsseldorf 1995, 789-791, 789, ist sie „ein bildlicher Ausdruck für einen Gegenstand (oft zur Veranschaulichung von abstrakten Bezeichnungen), einer Eigenschaft oder eines Geschehens". H. Vorgrimler, Neues Theologisches Worterbuch, Freiburg - Basel - Wien 2000, 415, versteht unter Metapher „eine Aussageweise, bei der ein bestimmter sprachlicher Ausdruck auf einen anderen gemeinten Ausdruck ubertragen wird". Ausfuhrlich zu den Metapherntheorien C.G. Muller, Gottes Pflanzung - Gottes Bau - Gottes Tempel. Die metaphorische Dimension paulinischer Gemeindetheologie in 1Kor 3,5-17 (FuSt 5), Frankfurt/M. 1995, 5-44.

10 H. Weinrich, a.a.O. 6.

${ }_{11}$ Ebd.

12 C.G. Müller, a.a.O. 23.

13 Ebd. 30. Vgl. H. Weinrich, Sprache in Texten, Stuttgart 1976, 283: „Im Maße, wie das Einzelwort in der Sprache keine isolierte Existenz hat, gehört auch die Einzelmetapher 
Diese weisen auf eine bodenbereitende „metaphorische Leitvorstellung ${ }^{\text {" } 14}$ hin, mit der „eine Potentialität von Metaphern gegeben ist“" ${ }^{\text {“5 }}$. Bei der Realisation einer Metapher kommt es zur „Koppelung zweier sprachlicher Sinnbezirke ${ }^{\text {"16 }}$, die man als „Bildspender“ und „Bildempfänger“ 17 kennzeichnet. Es ist dabei jedoch zu berücksichtigen, dass bestimmte Ausdrücke ihre metaphorische Bedeutung nur innerhalb des bestimmten Kontextes gewinnen ${ }^{18}$, wobei unter Kontext nicht nur „die unmittelbare literarische Umgebung“ zu verstehen ist, sondern auch „die konkrete Sprechsituation “19 und der „zeitgeschichtliche und sozio-kulturelle Rahmen“20.

Paulus bedient sich der Metaphern, weil er für seine Hörer bzw. Leser verständlich sein will. Es geht ihm dabei nicht um bloße Ornamente, sondern um

in den Zusammenhang eines Bildfeldes. Sie ist eine Stelle im Bildfeld“. Vgl. auch D. Peil, Untersuchungen zur Staats- und Herrschaftsmetaphorik in literarischen Zeugnissen von der Antike bis zur Gegenwart, München 1983, 24: „Als »Bildfeld “ wird... eine Struktur verstanden, die sich aus verschiedenen Bildelementen und den zwischen ihnen bestehenden Relationen zusammensetzt; Elemente wie Relationen können hinsichtlich ihrer Anzahl und Ausprägung variieren, so daß unterschiedliche Bildvarianten oder -auspragungen moglich sind".

14 D. Peil, a.a.O. 24.

15 C.G. Müller, a.a.O. 30. Vgl. auch H. Birus - A. Fuchs, Ein terminologisches Grundinventar für die Analyse von Metaphern, in: C. Wagenknecht (Hg.), Zur Terminologie der Literaturwissenschaft. Akten des IX. Germanistischen Symposions der Deutschen Forschungsgemeinschaft Würzburg 1986 (Germanische Symposien. Berichtbände; Bd. 9), Stuttgart 1988, 157-174, 162f: „Neben dem stärker oder schwächer konventionalisierten Charakter der einzelnen Metapher sind konventionelle Koppelungen von Begriffsbereichen (Bildfelder) zu beachten, in deren Rahmen "halbinnovative" Neuschöpfungen möglich sind".

16 H. Weinrich, Sprache 283.

17 C.G. Müller, a.a.O. 24. H. Weinrich, Sprache 284, kennzeichnet diese Koppelung „als bildspendendes und bildempfangendes Feld“.

18 So C.G. Müller, a.a.O. 30. Nach H. Weinrich, a.a.O. 370f, machen „Wort und Kontext... zusammen die Metapher“. Vgl. J. Zmijewski, Der Stil der paulinischen „Narrenrede“. Analyse der Sprachgestaltung in 2Kor 11,1-12,10 als Beitrag zur Methodik von Stiluntersuchungen neutestamentlicher Texte (BBB 52), Köln - Bonn 1978, 65: „In den meisten Fällen ist erst die Einbettung in den Satz- oder Textzusammenhang für den Bildcharakter der Wörter entscheidend“. Vgl. auch E.R. MacCormac, Die semantische und syntaktische Bedeutung von religiösen Metaphern, in: J.P. van Noppen (Hg.), Erinnern, um Neues zu sagen. Die Bedeutung der Metapher für die religiöse Sprache, Frankfurt am Main 1988, 84-107.

19 C.G. Müller, a.a.O. 30. Vgl. dazu G. Kurz, Metapher, Allegorie, Symbol, Göttingen 2 1988, 13: „So muß die metaphorische Bedeutung nicht als Eigenschaft einer Außerung bestimmt werden. Mit einer Äußerung ist eine kommunikative Situation gegeben, nach der erst entschieden werden kann, ob ein Ausdruck metaphorisch gemeint ist oder nicht". Nach W. Kugler, Zur Pragmatik der Metapher. Metaphernmodelle und historische Paradigmen, Frankfurt/M. - Bern - New York 1984, 1, ist die Metapher „in dem Rahmen zu sehen, in dem sie sich realisiert, d.h. im Rahmen der situierten Äußerung, des engeren und weiteren Kontexts".

20 C.G. Müller, a.a.O. 31. Nach H.J. Klauck, Allegorie und Allegorese in synoptischen Gleichnistexten, Münster ${ }^{2} 1986,145$, meint der „sozio-kulturelle Kontext... das epochale Wirklichkeitsmodell, das den umfassenden Horizont für die Verständigung des Autors mit seinen Adressaten angibt". 
den Inhalt seiner Botschaft, der nur so und nicht anders mitgeteilt werden kann (= „heuristische Valenz") $)^{21}$. In den metaphorischen Prädikationen des Apostels lässt sich schließlich „ein ausgeprägt paränetisches Interesse“ erkennen, d.h. er zielt darauf ab, bei seinen Hörern bzw. Lesern „Einstellungen zu bilden und Handeln zu leiten“ (= „paränetische Valenz" $)^{22}$.

\section{2. Ökologische Bildfelder in den paulinischen Briefen}

Paulus verwendet oft bildspendende Metaphern aus dem Bereich der Vegetation $^{23}$, z.B.: $\alpha \dot{u} \xi \alpha \dot{\alpha} \nu \in \iota \nu$ bzw. $\alpha$ uै $\xi \in L \nu$ - „wachsen“, „wachsen lassen“ (1Kor 3,6f; 2Kor 9,10; 10,15), карто́ - „Frucht“ (Röm 1,13; 6,21f; 1 Kor 9,7; Gal 5,22),

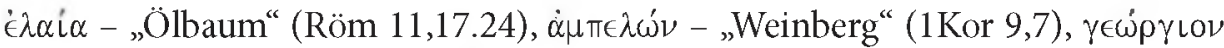

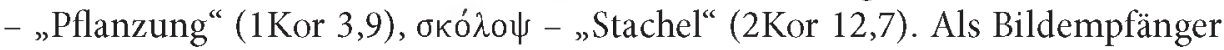
erscheint dabei in der Regel die Gemeinde (z.B. 1Kor 3,9b: „ihr seid Gottes

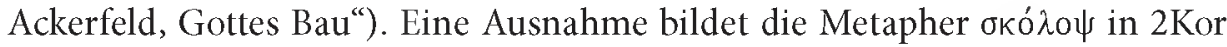
12,7, die Paulus auf sich selbst bezieht.

Andere bildspendende Metaphern stammen aus der Landwirtschaft ${ }^{24}$. Dazu

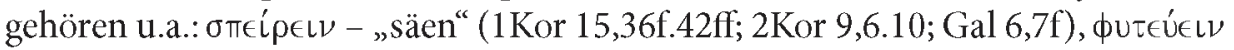

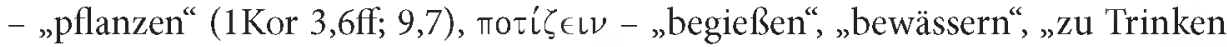

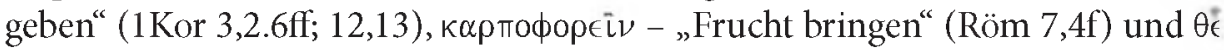

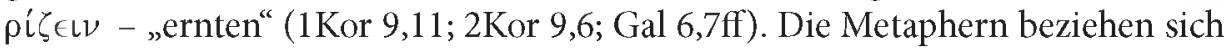
auf die Gemeinde (z.B. 1Kor 3,6: „Ich habe gepflanzt, Apollos hat begossen, Gott aber ließ wachsen") bzw. auf die Christen (Röm 7,4f).

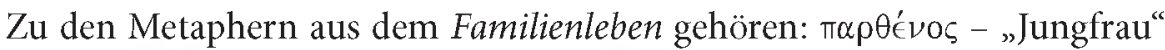

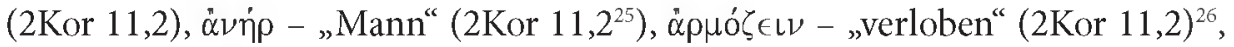

21 Nach C.G. Müller, a.a.O. 60, dient der metaphorische Sprachgebrauch „den Menschen, »etwas Charakteristisches an einer Person oder Sache" hervorzuheben, und verschafft auf diese Art und Weise Zugänge zur Wirklichkeit. Damit wird die Möglichkeit einer neuen Sicht der Wirklichkeit bereitgestellt. Mit dieser neuen Perspektive bringt die Metapher »auch Neues über die Wirklichkeit hervor «. Als heuristisches Instrument ist sie hilfreich, zu einem besseren Verständnis dessen, was ist, zu gelangen oder Neues bzw. Wahrgenommenes verstehbar zu machen“.

22 So C.G. Müller, a.a.O. 61. Vgl. E. Jüngel, Metaphorische Wahrheit. Erwägungen zur theologischen Relevanz der Metapher als Beitrag zur Hermeneutik einer narrativen Theologie (1974), in: Ders., Entsprechungen: Gott - Wahrheit - Mensch. Theologische Erörterungen (BevTh 88), München 1980, 103-157, 153: „Metaphern sprechen an und sollen ansprechen“.

23 Vgl. P. von Gemünden, Vegetationsmetaphorik im Neuen Testament und seiner Umwelt. Eine Bildfelduntersuchung (NTOA 18), Freiburg - Göttingen 1993, 266-289; ferner G.B. Ladner, Handbuch der fruhchristlichen Symbolik. Gott, Kosmos, Mensch, Stuttgart - Zürich 1992, $130 \mathrm{ff}$.

24 Vgl. P. von Gemünden, a.a.O. 289-310.

25 Vgl. zu dieser Stelle J. Zmijewski, a.a.O. 80-85.

26 Ausführlich dazu R. Zimmermann, Geschlechtermetaphorik und Gottesverhältnis. Traditionsgeschichte und Theologie eines Bildfelds in Urchristentum und antiker Welt (WUNT II/122). Tübingen 2001, 300-325. 


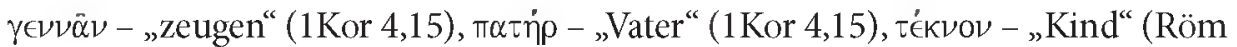
8,16f.21; 9,7f; 1Kor 4,14.17; 2Kor 6,13), viós - „Sohn“ (Röm 8,14.19; Gal 3,7.26;

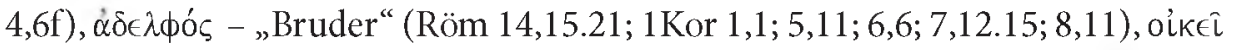

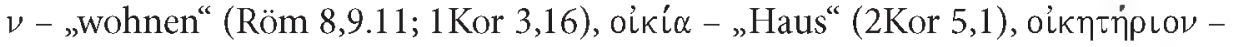

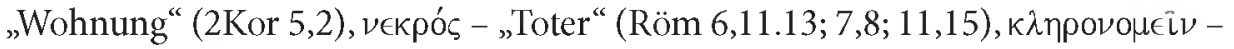

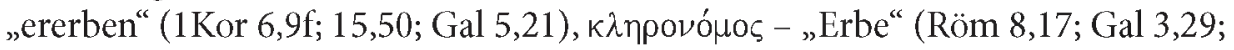

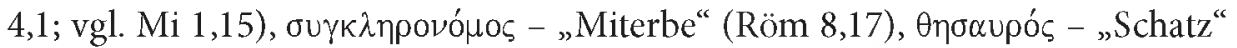

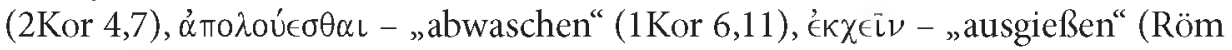

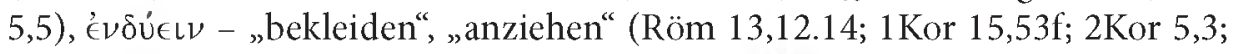

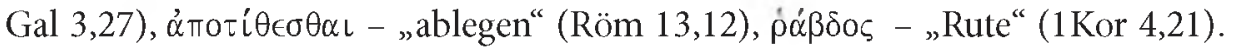
Bildempfänger sind auch hier in erster Linie die Gemeinde bzw. die Christen (z.B. 2Kor 5,1: „Wir wissen: Wenn unser irdisches Zelt abgebrochen wird, dann haben wir eine Wohnung von Gott, ein nicht von Menschenhand errichtetes ewiges Haus im Himmel“), in wenigen Fällen beziehen sich die Metaphern aber auch auf Gott (Röm 5,5) oder den Apostel (1Kor 4,15.21; 2Kor 11,2).

Der Apostel benutzt ferner Metaphern aus der Arbeitswelt, z.B. diese:

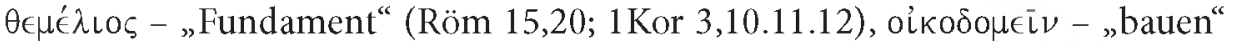

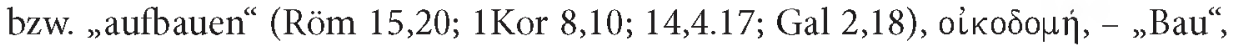
"Erbauung", „Aufbau“ (1Kor 3,9; 14,3.5.12.26; 2Kor 10,8; 12,19; 13,10) ${ }^{27}$,

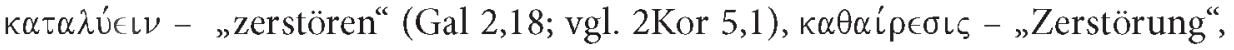

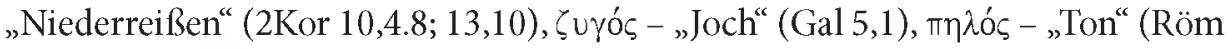

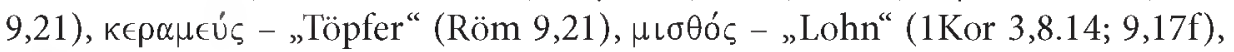
$\sigma \tau U ̂ \lambda o \varsigma$ - „Säule“ (Gal 2,9). Als Bildempfänger erscheinen dabei Paulus (z.B. 1Kor 3,10: „Der Gnade Gottes entsprechend, die mir geschenkt wurde, habe ich wie ein guter Baumeister das Fundament gelegt; ein anderer baut darauf weiter. Aber jeder soll darauf achten, wie er weiterbaut"; ferner Röm 15,20; 1Kor 3,8f; 4,9; 8,$10 ; 9,17 f ; 14,3 f f .12 .17 .26$; 2 Kor 10,4.8; 12,9; 13,10; Gal 2,18), die Christen bzw. Gemeinden (Röm 9,21; Gal 5,1), der Leib des Christen (1Kor 6,19), die Apostel Jakobus, Kephas und Johannes (Gal 2,9), Gott (Röm 9,21).

\section{Zu den ökologischen Aufgaben des Apostels}

Wie oben gezeigt wurde, bedient sich Paulus der ökologischen Metaphern sowohl in Bezug auf die Gemeinde (z.B.: 1Kor 3,9b: „ihr seid Gottes Ackerfeld, Gottes Bau“) als auch auf apostolische Aufgaben (z.B.: 1Kor 3,6: „Ich habe gepflanzt, Apollos hat begossen, Gott aber ließ wachsen"). Die Tatsache, dass Paulus sich als der mit der Gnade (Röm 1,5; 12,3; 15,15; 1Kor 2,10; 3,10; 7,40;

\footnotetext{
Zum Bildfeld „Bauen“ vgl. u.a. I. Kitzberger, Bau der Gemeinde. Das paulinische Wortfeld

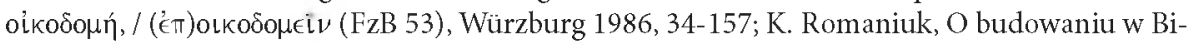
blii, Warszawa 1988, 54-73.
} 
15,10; Gal 1,15f; 2,9) und Vollmacht (Röm 15,19; 2Kor 6,7; 10,8; 12,9; 13,10) ausgerüstete „Stellvertreter“ Christi (2Kor 5,20) weiß, erklärt, warum er „ökologische Aufgaben“, die im Alten Testament Gott und im Neuen Testament Jesus Christus zugeordnet werden, auf seine eigene Sendung beziehen kann. Als Beispiel ist die Metapher vom „Baumeister“ zu nennen. Schon bei den alttestamentlichen Propheten begegnet das Bildfeld vom Bauen ${ }^{28}$. Als Baumeister erscheint dabei in der Regel Gott selbst. Er ist der, der sein Volk „aufbaut“ (Jer 1,$10 ; 18,9 ; 24,6 ; 31,4.28 ; 33,7 ; 42,10 ;$ vgl. auch Jes 62,5; Jer 12,15ff; Am 9,11) bzw. ein Fundament legt ${ }^{29}$ (vgl. Jes 28,16$)^{30}$. Dieses im Alten Testament vorgeprägte Bildfeld wendet Paulus auf sich an, wenn er sich z.B. in 1 Kor 3,10 als бoфò

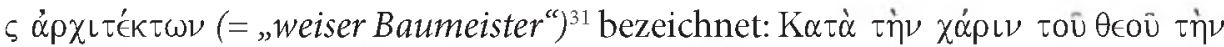

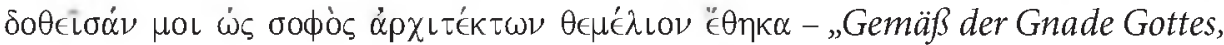
die mir gegeben ist, habe ich wie ein weiser Baumeister ein Fundament gelegt". Mit dieser Selbstbezeichnung will er vielleicht zum Ausdruck bringen, dass Israel

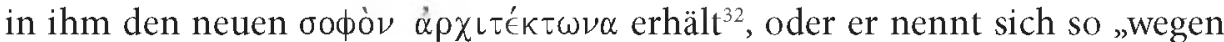
des ihm vorgeworfenen Mangels an Weisheit... und im Blick auf die bildhaft als

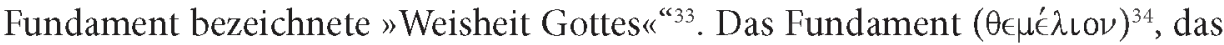
er als „weiser Baumeister" gelegt hat, ist Jesus Christus $(3,11)^{35}$. Mit diesem Bild vom Legen des entscheidenden Fundaments in der Gemeinde ${ }^{36}$ hebt der Apostel

28 Vgl. dazu Ph. Vielhauer, Oikodome. Das Bild vom Bau in der christlichen Literatur vom Neuen Testament bis Clemens Alexandrinus (1939), in: Ders., Oikodome. Aufsätze zum Neuen Testament. Bd. 2, hg. von G. Klein (TB 65), München 1979, 1-168, 4-8.

29 Vgl. C.G. Müller, a.a.O. 85.

30 J. Lindblom, Der Eckstein in Jes. 28,16, in: NTT 56(1955), 123-132, 126, hebt den metaphorischen Charakter des Textabschnitts von Jes 28,16 hervor und stellt fest: „Es handelt sich um eine Schöpfung Jahwes unter dem Bild eines Gebäudes, von dessen Bestandteilen besonders der Gründungseckstein und die Mauern erwähnt werden".

31 Das Wort $\dot{\alpha} \rho \chi\left\llcorner\tau \tau^{\prime} \in \tau \omega \nu\right.$ ist hapax legomenon im Neuen Testament.

32 So H. Hübner, Biblische Theologie des Neuen Testaments, Bd. II: Die Theologie des Paulus und ihre neutestamentliche Wirkungsgeschichte, Göttingen 1993, 131 Anm. 282: „Nach Jes 3,1ff. straft Gott Jerusalem und Juda, indem er ihnen alle die Männer nimmt, die sie zu ihrer Existenz brauchen, auch den "weisen Architekten «. Sollte, was wahrscheinlich ist, Paulus bei der Formulierung von 1Kor 3,10 diese Jes-Stelle vor Augen gehabt haben, so ist zu fragen, ob er mit der verbalen Anleihe an sie zum Ausdruck bringen wollte, daß Gott durch seine Berufung diesen Architekten dem nun neuen Israel in der Gestalt des Apostels zurückgegeben hat. So wenig dies beweisbar ist, so sehr fügt sich jedoch eine derartige Annahme in das apostolische Selbstbewußtsein des Paulus, auch und gerade, wie es sich in 1 Kor 3 artikuliert“.

33 J. Kremer, Der Erste Brief an die Korinther (RNT), Regensburg 1997, 73.

34 Zum Begriff vgl. K.L. Schmidt, Art. $\theta \in \mu$ é $\lambda$ Lo $\kappa \tau \lambda$., in: ThWNT III, 63-64.

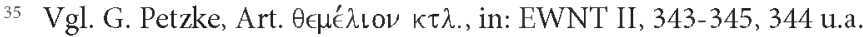

36 Nach J. Roloff, Apostolat - Verkündigung - Kirche. Ursprung, Inhalt und Funktion des kirch-lichen Apostelamtes nach Paulus, Lukas und den Pastoralbriefen, Gütersloh 1965, 105, ist die Grundlegung des Fundaments „das beherrschende Bild im Bereich der ekklesiologisch orientierten Selbstaussagen des Apostels“. 
die „fundamentale“ Bedeutung seines Wirkens und damit auch seine „Autorität und Sonderstellung ${ }^{“ 37}$ ihr gegenüber hervor. Wenn er dabei die Baumetaphorik durch die Vegetationsmetaphorik ergänzt (vgl. 3,6-9), dann ist diese Verbindung schon im Alten Testament, besonders bei den Propheten zu finden (2Sam 7,10; Jes $5,1 f ; 58,11 f ; 61,3 f ; 65,21 f ;$ Jer 1,$10 ; 18,9 ; 24,6 ; 29,5 ; 31,28 ; 42,10 ; 45,4$; Ez 28,26; 36,9f.36; Am 9,11.14f) $)^{38}$.

Die Baumetaphorik begegnet unter einem anderen Aspekt auch innerhalb des sog. "Kampfbriefes“ des zweiten Korintherbriefes (Kap. $10-13$ ) ${ }^{39}$. So

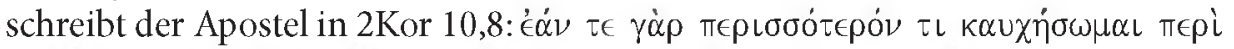

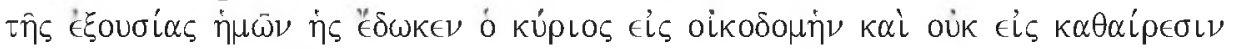

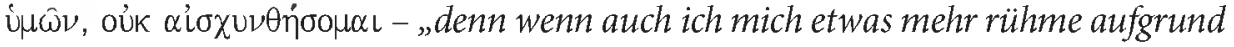
der Vollmacht, die der Herr uns zu eurem Aufbau und nicht zu eurer Zerstörung gegeben hat, werde ich nicht zuschanden werden". Bei der Verteidigung seines Apostolats gegenüber seinen Gegnern macht Paulus klar, dass er sich größerer Christusnähe als sie rühmen könnte ${ }^{40}$, und weist dafür auf seine besondere apostolische „Vollmacht“ ( $\dot{\epsilon} \xi$ ou $\sigma i \alpha)^{41}$ hin $^{42}$. Diese Vollmacht „ist ihm Gegenstand besonderen »Rühmens«..., da sie nicht eine sich selbstbeigelegte Autorität besagt, sondern Fortsetzung der Vollmacht des erhöhten Herrn für die Kirche ist “43. Seine Vollmacht ist also kein eigenes Verdienst, sondern sie wurde ihm vom кúpı geschenkt und dient „zum Aufbau und nicht zur Zerstörung“ (

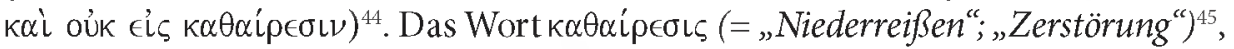

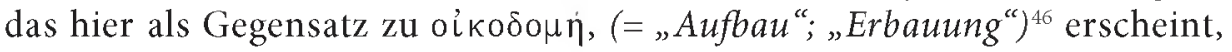

37 Ph. Vielhauer, a.a.O 80.

38 Vgl. Ph. Vielhauer, a.a.O. 7: „Das Bild vom Pflanzen ist dem vom Bauen ganz parallel, formal dadurch, daß es häufig mit seinem Gegensatz »ausreißen « auftritt und dann meist in Verbindung mit "bauen - niederreißen ", inhaltlich dadurch, daß es sich in seiner Bedeutung mit dem Bild vom Bau deckt (so in... Jeremiastellen 1,10; 24,6; 31,27f; 42,10; 45,4 und Ez 36,36)“.

39 Zu den im jetzigen 2. Korintherbrief enthaltenen Einzelschreiben vgl. A.J. Najda, a.a.O. 149.

40 Vgl. H.J. Klauck, 2. Korintherbrief (NEB.NT 8), Würzburg 1986, 79: „Streitpunkt ist... ein besonderes Dienstverhältnis zu Christus, in dem für Paulus sein Apostel-Sein gründet“. E. Walter, Der zweite Brief an die Korinther (GSL.NT 7), Düsseldorf 1964, 77, spricht von einer „Christusbeziehung ganz anderer Art“.

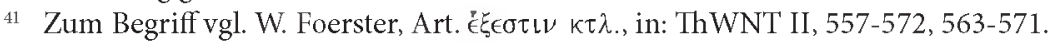

42 Vgl. C. Wolff, Der zweite Brief des Paulus an die Korinther (ThHK 8), Berlin 1989, 201: „Seine apostolische Vollmacht ist größer als die - angemaßte - seiner Gegner, er kann sich ihrer in besonderer Weise rühmen und wird dann nicht etwa als ein Lügner beschämt werden“.

43 F. Zeilinger, Krieg und Friede in Korinth. Kommentar zum 2. Korintherbrief des Apostels Paulus. Teil I: Der Kampfbrief. Der Versöhnungsbrief. Der Bettelbrief, Wien - Köln - Weimar 1992, $49 \mathrm{f}$.

44 Vgl. U. Heckel, Kraft in Schwachheit. Untersuchungen zu 2.Kor 10-13 (WUNT II/56), Tübingen 1993, 14.

45 Zum Begriff $\kappa \alpha \theta \alpha i ́ \rho \epsilon \sigma \iota \varsigma$ vgl. C. Schneider, Art. $\kappa \alpha \theta \alpha \iota \rho \epsilon \omega \kappa \tau \lambda$., in: ThWNT III, 414-416.

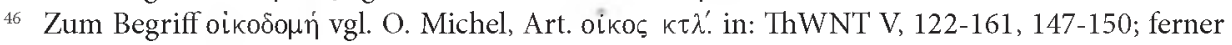
I. Kitzberger, a.a.O. 34-157. 
bezieht sich wohl auf die Gegner, welche im Unterschied zu ihm die Gemeinde zerstören, statt sie aufzubauen ${ }^{47}$. Wenn Paulus sich demgegenüber als einer weiß, der zum "Aufbau“ der Gemeinde wirkt, dann lehnt er sich „an prophetisches Selbstverständnis“" ${ }^{48}$ an. Die Redeweise von „Aufbau und Zerstörung“ ist deutlich eine Anspielung auf alttestamentliche Propheten, bei denen sie häufig begegnet. Neben Jes 49,17; Jer 31,28; 42,10; 45,4; Ez 36,36; Mal 1,4 sind vor allem Jer 1,10 und 24,6 zu nennen ${ }^{49}$. Nach Jer 1,10 sind Aufbauen und Zerstören "Auftrag Gottes an den Profeten ${ }^{\times 50}$, während sie sonst im Jeremiabuch $(31,3.28 ; 42,10 ; 45,4)$ als „Tat Gottes selber ${ }^{\text {“51 }}$ erscheinen. Paulus übernimmt die prophetische Aufgabe des "Aufbaus“ der Gemeinde. Allerdings nur diese; das „Niederreißen“ dagegen bleibt für ihn ein „opus alienum “52. Sein „Aufbau“ geschieht dabei im Sinne

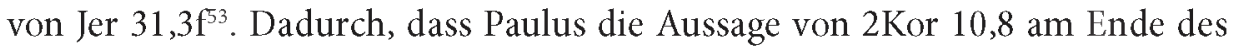
„Kampfbriefes", näherhin in 13,10, fast wörtlich wiederholt - wodurch der ganze Brief eine bezeichnende Rahmung erhält ${ }^{54}$-, unterstreicht er die Bedeutung, die dem „Aufbau“ der Gemeinde in seinem Selbstverständnis zukommt.

\section{Fazit}

Von der Ökologie ist oft in der Heiligen Schrift die Rede. Schon bei der Erschaffung der Welt bekommen die Menschen einen Befehl von Gott dem Schöpfer: „, Seid fruchtbar, und vermehrt euch, bevölkert die Erde, unterwerft sie euch, und herrscht über die Fische des Meeres, über die Vögel des Himmels und über alle Tiere, die sich auf dem Land regen" (Gen 1,28) ${ }^{55}$. Die Menschen aller Zeit antworten auf den göttlichen Befehl durch ihr Handeln in der Welt. Diese der die ganzen Menschheit anvertraute „ökologische Aufgabe“ ist sowohl Paulus selbst als auch seinen Gemeinden bewusst, verständlich und wichtig. Deshalb bedient sich der Apostel gern und relativ häufig der ökologischen Metaphern nicht nur dann, wenn er die Wirklichkeit der christlichen Gemeinde, ihre Natur

47 Vgl. dazu C. Schneider, ThWNT III, 416.

48 H.J. Klauck, a.a.O. 79.

49 Vgl. J. Zmijewski, Paulus - Knecht und Apostel Jesu Christi. Amt und Amtsträger in paulinischer Sicht, Stuttgart 1986, 183 Anm. 217; H. Rusche, Zum "jeremianischen " Hintergrund der Korintherbriefe, in: BZ NF 31(1987), 116-119, 118. Nach C. Wolff, a.a.O. 201, ist daraus allerdings „ein an Jeremia anknüpfendes Selbstverständnis des Paulus... nicht zu erschließen“.

${ }_{50} \mathrm{Ph}$. Vielhauer, a.a.O 73.

51 Ebd.

52 Ebd.

53 So wie dort (Jer 31,3f) Gott Israel „erbaut", indem er seine Liebe schenkt, baut Paulus die Gemeinde aus Liebe zu ihr (1Kor 4,21; 16,24; 2 Kor 2,4; 11,2.11; 12,15) auf. Vgl. H. Rusche, a.a.O. 118.

54 Vgl. F. Zeilinger, a.a.O. 50; C. Wolff, a.a.O. 201 u.a.

55 Vgl. dazu A.J. Najda, Początki ekologii w Biblii, in: Studia Ecologiae et Bioethicae 2(2004), 143 -150 . 
und Aufgaben zum Ausdruck bringen will, sondern auch seine missionarische Tätigkeit und sein apostolisches Selbstverständnis.

\title{
O ekologicznych metaforach u Pawła
}

\section{STRESZCZENIE}

Paweł wielokrotnie w swoich listach podkreśla, że jest apostołem Chrystusa Jezusa wybranym, powołanym i posłanym do głoszenia Ewangelii Bożej (por. Rz 1,1; 1 Kor 1,$1 ; 2$ Kor 1,1). W swoim nauczaniu apostoł posługuje się chętnie różnymi metaforami, wśród których ważną rolę odgrywają metafory z zakresu ekologii. Paweł wykorzystuje je wtedy, gdy mówi o rzeczywistości, naturze i zadaniach gminy chrześcijańskiej (np. $\mathrm{Rz} 7,4 \mathrm{n} ; 9,21$; 1 Kor 3,9), gdy chce opisać swoją działalność misyjną lub wyrazić swoją apostolską świadomość (np. 1Kor 3,6; 3,10; 2 Kor 10,8; 13,10).

\section{Ecological Metaphors in the Writings of St. Paul}

\author{
SUMMARY
}

Many times in his letters Paul underlines that he is an apostle of Christ Jesus chosen, called and sent to proclaim the Gospel of God (cfr. Rom 1,1; 1 Cor 1,1; 2 Cor 1,1). In his teaching he willingly used different metaphors, among which those taken from the field of ecology had an important role. Paul makes use of them when speaks of the reality, the nature and the tasks of the Christian community (e.g.: Rom 7,4ff.; 9,21; 1 Cor 3,9), when he wants to describe his missionary activity or to express his apostolic consciousness (e.g.: 1 Cor 3,$6 ; 3,10 ; 2$ Cor 10,$8 ; 13,10$ ). 